\title{
Anatomic Site Distribution of Colorectal Cancer: A Retrospective Study of 102 Cases in a Tertiary Hospital
}

\author{
M A Alim ${ }^{1}$, M H Rashid ${ }^{2}$, M A Ahad ${ }^{3}$, M M H chowdhury ${ }^{4}$, M K Rahman 4 , \\ M M R Khan ${ }^{4}$, M Azizul Haque $^{4}$
}

\begin{abstract}
Colorectal carcinoma is one of the major cancers in the developed world. It is not uniformly distributed through out the large bowel. Recent studies of patients with colorectal cancer have suggested a shift towards the proximal colon and an increase in incidence of right sided colon cancer. This study aimed to determine the anatomical distribution of colorectal in our tertiary level hospital. A total of 102 patients were selected retrospectively from Department of Gastroenterology in Rajshahi Medical College. Records of patients of colorectal cancer were reviewed for demographic data, histological type and anatomical location of tumour. Tumour located at or proximal to splenic flexure were defined as right sided cancer and tumour arising distal to the splenic flexure were defined as left sided cancer. Out of 102 patients, 74 are male and 28 are female and male: female ratio 2.6:1. The age range was from 21 to 71 years with mean 46.5 \pm 13.3 . Approximately seventy eight percent of cancer was left sided and $21 \%$ were right sided. Age and sex related distribution of proximal and distal tumors were analyzed but no statistically significant relation has been observed. So it is observed that the anatomical distribution of colorectal cancer has been fairly constant with no evidence of proximal shift of colorectal cancer. However multicenter large volume studies can bring a concrete opinion.
\end{abstract}

TAJ 2010; 23(1): 22-25

\section{Introduction}

Colorectal cancer (CRC) is the most common gastrointestinal malignancy throughout the developed world and the second leading cause of cancer death in the united states ${ }^{1,2}$. Despite of slight decrease in its incidence and mortality during past two decades approximately 140000 people are affected annually and about 50000 cancer related death per year ${ }^{3}$.The incidence of colorectal cancer is considered low in India. But in recent years due to westernization of life styles and nutritional habit, the rate is increasing.(4).Colorectal cancer is not uniformly distributed throughout the large bowel. In General two-thirds of colorectal malignancies are localized in the left colon and rectum.

During last two decades many recent studies have depicted an anatomic shift of colorectal cancer toward right side of colon ${ }^{5}$.Some investigators using age- specific and sex specific analysis methods have drawn the conclusions that patients with right sided cancer are older than those with distal colorectal cancer. Women made up a higher percentage of patient with proximal colon cancers $^{6,7,8}$. So this study is aimed to determine the anatomical site distribution of colorectal cancer in tertiary hospital.

${ }^{1}$ Associate Professor, Department of Gastroenterology, Rajshahi Medical College, Rajshahi.

${ }^{2}$ Assistant Professor, Department of Hepatolgy, Rajshahi Medical College, Rajshahi.

${ }^{3}$ Associate Professor, Department of Gastroenterology, Khulna Medical College, Khulna.

${ }^{4}$ Assistant Professor, Department of Medicine, Rajshahi Medical College, Rajshahi. 


\section{Material and Methods}

A retrospective study was undertaken. Consecutive 102 patients were enrolled from colonoscopy patients' registrar in the Department of Gastroenterology of Rajshahi Medical College Hospital. The study period was March 2007 to Feb2010. Data were reviewed \& age, sex and tumour location were recorded for each case. All patients are histologically proven colorectal adenocarcinoma and anatomic sites of involvement were assessed with full colonoscopy. Patients with synchronous cancer, inflammatory bowel disease and familial adenomatous polyposis were excluded from this study. Tumour located at or proximal to splenic flexure was defined as right sided cancer and tumour arising distal to the splenic flexure were defined as left sided cancer ${ }^{9}$.

Data were analyzed with statistical package for social science (SPSS) 12.0 program. Variables were recorded as descriptive frequencies. Differences were reported as statistical significant if the $\mathrm{p}$ value was less than0.05. Data are expressed as mean, standard deviation and 95\% CI (confidence interval) .Statistical analysis was performed using chi-square $\left(\chi^{2}\right)$ are given in SPSS.

\section{Results}

All 102 patients were included in this study, of which 74 are male and 28 are female and male: female ratio 2.6:1. The age range was from 21 to 71years with mean 46.5 \pm 13.3 . Clinicodemographic profiles have been described in table 1. All patients had abdominal pain or discomfort and also had some sort of bowel alteration. Majority of patients (73.5\%) were with rectal bleeding. Distal colon cancer including rectal cancer was overall still common \& comprising (78.4\%). Among distal cancer recto-sigmoid is the most predominant location (26.4\%) (Table2).Our study revealed proximal cancer is low (21.5\%) and did not show any right sided shifting (Table 2). The distribution of colorectal cancer according to age and sex were shown in Table $3 \& 4$ and no statistically significant relation with either age or sex was observed. Histologically all patients were with adenocarcinoma.
Table 1: Clinico-demographic characteristic of patients with carcinoma colon $(\mathrm{n}=102)$

\begin{tabular}{ll}
\hline Age (yr) & $46.5 \pm 13.3$ \\
Sex (male:female) & $2.6: 1$ \\
Residence & \\
Rural & $64(62.7 \%)$ \\
Urban & $38(37.3)$ \\
Social status & \\
Poor( <5000/- Tk/ mon) & $43(42.2 \%)$ \\
Middle (5000-10000/-Tk./mon) & $51(50.0 \%)$ \\
Upper class $>10000 /-T k . / m o n)$ & $8(7.8 \%)$ \\
Positive Family history of colon cancer & $2(1.96 \%)$ \\
Abdominal pain & $102(100 \%)$ \\
Bowel habit & \\
Loose - & $78(76.5 \%)$ \\
Constipation- & $8(7.8 \%)$ \\
Mixed & $16(15.7 \%)$ \\
Rectal bleeding & \\
Present & $74(73.5 \%)$ \\
Absent & $28(27.5 \%)$ \\
Abdominal lump & \\
Present & $38(37.3 \%)$ \\
Absent & $64(62.7 \%)$ \\
Anemia & \\
Mild- & $11(10.8 \%)$ \\
Moderate & $60(58.8 \%)$ \\
Severe & $31(30.4 \%)$ \\
\hline
\end{tabular}

Table 2: Distribution of Colorectal cancer $(n=102)$.

\begin{tabular}{ll}
\hline Location & No (percent) \\
Rectum & $22(21.5 \%)$ \\
Colon & $80(78.4 \%)$ \\
Proximal & $22(21.5 \%)$ \\
Cecum & $12(11.7 \%)$ \\
Colon ascends & $8(7.8 \%)$ \\
Transverse colon & $2(1.9 \%)$ \\
Distal & $58(56.8 \%)$ \\
Colon descends & $14(13.7 \%)$ \\
Sigmoid & $17(16.6 \%)$ \\
Recto-sigmoid & $27(26.4 \%)$ \\
\hline
\end{tabular}

Table 3: Relation between anatomical distribution with age $(n=102)$.

\begin{tabular}{lllll}
\hline \multicolumn{1}{c}{ Age } & \multicolumn{2}{c}{ Distribution of location } & Total & P value \\
\cline { 2 - 3 } & \multicolumn{1}{c}{ Proximal } & Distal & & \\
\hline$\leq 40$ years & $6(5.8 \%)$ & $27(26.4 \%)$ & $33(32.3 \%)$ & \\
$>40$ years & $16(15.6 \%)$ & $53(51.9 \%)$ & $69(67.6 \%)$ & $\chi 2=0.76$ \\
Total & $22(21.5 \%)$ & $80(78.4 \%)$ & $102(100 \%)$ & \\
\hline
\end{tabular}

Table 4: Anatomic distribution according to sex $(n=102)$

\begin{tabular}{llcll}
\hline Sex & \multicolumn{2}{c}{ Distribution of location } & Total & P value \\
\cline { 2 - 4 } & \multicolumn{1}{c}{ Proximal } & Distal & & \\
\hline Male & $17(16.6 \%)$ & $57(55.8 \%)$ & $74(72.5 \%)$ & \\
Female & $5(4.9 \%)$ & $23(22.5 \%)$ & $28(27.4 \%)$ & $\chi 2=0.57$ \\
Total & $22(21.5 \%)$ & $80(78.4 \%)$ & $102(100 \%)$ & \\
\hline
\end{tabular}




\section{Discussion}

Colorectal carcinoma is the second most common cancer in the western hemisphere of the world and very important cause of mortality and morbidity. Colorectal carcinoma is not uniformly distributed through out the large bowel. Compared with western countries, the incidence of CRC is low in Asia, but the dietary habits and life styles have influenced greatly the increasing incidence in developing area of world. Rectal and sigmoid cancer accounts for a disproportionately high percentage of cancers in large bowel. The actual mechanism is not clear. Rates of CRC also vary considerably with geography. In recent years, there are some changes in pattern of CRC, such as increasing incidence rate in Japan, Eastern Europe and China with continued development of right ward shift of CRC along with decrease of rectal cancer. ${ }^{10,11,12,13,14}$. Analysis of our data disclosed that the distribution pattern of CRC is quite stationary.

This rightward shift may be related to improved diagnostic accuracy and varying etiological factors. There may several potential causes for proximal shift of colorectal cancer. . The proximal and distal colon has different embryologic origins, morphology, physiology and functions. Caecum, colon ascends, and proximal two- third of transverse colon derives from mid-gut, whereas the segment comprising the splenic flexure to upper anal canal derived from hind gut. The distinct embryologic origin of each segment are reflected the dual blood supply of normal colon. The proximal colon is primarily involved in water absorption and solidification of fecal content but the distal colon primarily for storage. The metabolic pathways such as that of glucose, butyrates and polyamines are also different .It has therefore been hypothesized that the proximal and distal colon are different organ. It may mean differences in differential sensitivities and exposure to carcinogens for the proximal and distal section of colon and rectum ${ }^{15}$.

In low risk countries, rectal cancer accounts for the largest proportion of all colorectal cancer ${ }^{16}$.
Our study findings are making this conformity well. The proportion of rectal cancer among all colorectal cancer is generally less than $40 \%$ in Europe and North America, in contrast to $50 \%$ or more in Asia ${ }^{17}$. In our study rectum and rectosigmoid cancer is the commonest site of involvement in Bangladesh.

Several studies reported the predominance of proximal cancer among female patient and in advanced age in developed countries ${ }^{18,} 19$. The reasons for age and sex difference, however remains unclear. Risk factors for CRC include genetic and environmental factors such as obesity, insufficient physical activity, alcohol and red meat consumption. Hormone replacement therapy and non-steroidal anti-inflammatory drugs use have a protective effect. ${ }^{20}$ We have analyzed the age and sex related distribution of proximal and distal tumors but no statistically significant relation have been observed.

So in conclusion the anatomical distribution of colorectal cancer has been fairly stable in this hospital with no evidence of proximal shift of colorectal cancer. However multi-center large volume studies can bring a concrete opinion.

The limitation of this study was that it comprised small number of sample and location has been determined only by colonoscopic means and indeed there is no precise landmark for colonic segment identification by colonoscopic procedure.

\section{References:}

1. Ries LA, Wingo PA, Miller DS, Howe HL, Weir HK, Rosenberg HM,Vernon SW, Cronin K, Edward Bk. The annual report to the nation on the status of cancer, 1973-1997, with a special section on colorectal cancer. Cancer 2000;88:2398-2424.

2. Greenlee Rt, Murray T, Bolden S, Wingo PA. Cancer statistics, 2000.CA cancer J Clin2000;50:7-33.

3. Greenle RT, Hill- Harmon MB, Murray T, Thun M. Cancer statistics 2001. Ca cancer J clin2001;51:15-36.

4. Ming Li, Jin Gu. Chaging patterns of colorectal cancer in China over perod of 20 years. World juonal gastroenterol2005; 11(30):4685-4688).

5. Greene FL. Distribution of colorectal neoplasm. A left to right shift of polyps and cancer. Ann surg 1983; 49; 62-65. 
6. Ming JS, Wong JM, Wang CY. Site distribution of colorectal caner: A retrospective study of 1198 cases. Chinese journal gastroenterol1990; 7:116121

7. Alley PG, McNee RK. Age and sex differences in the right colon cancer. Dis Colon Rectum1986; 29:227-229.

8. Golematis BG, Tzardis PJ, Ahwal JA et al. Site distribution of carcinoma of large intestine:retrospective study of 600 cases. Dis Colon Rectum 1989; 32:14-16.

9. Qing $\mathrm{SH}$, Rao KY, Jiang HY, Wexner SD. Racial differences in the anatomical distribution of Colorectal cancer:A study of differences between American and Chinese patients.World $\mathrm{J}$ gastroenterol2003;9:721-725.

10. LandsWe, Hamazaki T, Yamazaki K,Okuyama H,Sakai K, Goto Y, Hubbard VS. Chnaging dietary pattern. Am J ClinNutr1990; 51:195-202.

11. Levin KE,Dozols RR. Epidemiology of large bowel cancer. World J surgery; 1991:15:562-\%67.

12. Wilmink AB. Overview of epidemiology of colorectal cancer. Dis. Colon rectum 1997; 40:483-493.

13. Morgenstein L, Lee Se. Spatial distribution ofcolonic carcinoma. Arch Surg1978; 113:1142-1143.
14. Beart RW, Meltom LJ, Maruta M, Dockerty MB, Frydenberg HB, O'dallon WM. Trend in right and left sided colon cancer.

15. Bufll JA. Colorectal cancer:evidence if disatinct genetic categoriesbased on proximal and distal tumour location.Ann intern med1990;113779-788.

16. Waterhouse JA, Muir CS,Shanmugaratnam $K$, PowellJ. IARC scientific publication No42.International agency for research on cancer:Lyon 1982.

17. Devesa SS, Chow WH. Variation in colorectal cancer incidence In the United States By subsite of origin > Cancer1993; 71:3819-3826.

18. Bray F, Larsan IK. Trend in colorectal cancer in Norway: an interptretation of temporal pattern by anatomic subsite. Int J cancer2010; 126:721-732.

19. Wu XC, Chen VW, Steele B, Ruiz B, Fulton J, Liu L et al. Subsite specific incidence rate andstage of cdisease in corectal cancer by race gender and agegroupin united states. Cancer2001; 92:25472554.

20. Giovannucci E, Wu K. Cancer of colon and rectum. In;SchottenfeldD, FaunmeniJf, Jr.editors. Cancer epidemiology and prevention. $3^{\text {rd }}$ ed. New York: Oxford University Press; 2006; 809-829. 